\title{
Adriamycin loading and release characteristics of albumin-heparin conjugate microspheres
}

\author{
H.F.M. Cremers ${ }^{\mathrm{a}}$, R. Verrijk ${ }^{\mathrm{b}}$, H.P.J.M. Noteborn ${ }^{\mathrm{b}}$, G. Kwon ${ }^{\mathrm{c}}$, Y.H. Bae ${ }^{\mathrm{c}}$, S.W. Kim ${ }^{\mathrm{c}}$ and \\ J. Feijen ${ }^{\mathrm{a}, *}$ \\ ${ }^{a}$ Department of Chemical Technology, University of Twente, P.O. Box 217, 7500 AE Enschede, The Netherlands; \\ 'Division of Experimental Therapy, The Netherlands Cancer Institute, Amsterdam, The Netherlands; \\ ${ }^{c}$ Department of Pharmaceutics \& Center for Controlled Chemical Delivery, University of Utah, Salt Lake City, Utah, USA
}

(Received 14 May 1993; accepted 13 September 1993)

Biodegradable ion-exchange microspheres, prepared from a prefabricated conjugate of albumin and heparin were investigated as carriers for adriamycin. The ion-exchange microspheres could be loaded with adriamycin giving payloads up to $33 \% \mathrm{w} / \mathrm{w}$, depending on the heparin content of the conjugate. In vitro adriamycin release depended on the ionic strength of the release medium. In ion containing media, for instance saline, $90 \%$ of the drug was released within $45 \mathrm{~min}$, whereas in non-ionic media, such as distilled water, only $30 \%$ was released. Drug release profiles could be modelled by combining ion-exchange kinetics and diffusion controlled drug release models.

Key words: Adriamycin; Microsphere; Ion-exchange; Hydrogel; Drug delivery

\section{Introduction}

Cytostatic agents are widely used for the treatment of cancer. In general these drugs have narrow therapeutic windows. Due to the high systemic levels of the drug needed to achieve sufficiently high drug levels at the tumour site, severe side effects can occur. Adriamycin (ADR), for instance, is known to cause cardiomyopathy $[1,2]$. A reduction of the side effects can be achieved by targeting the drug to the site of the tumour, thus reducing systemic drug levels and confining the cytotoxicity of the drug to the tumour.

Microspheres are suitable carriers to be used for drug targeting [3,4]. Administration of drug-

*Corresponding author. loaded particles of the requisite size $(15-60 \mu \mathrm{m})$, may cause tumour infarction by occlusion or induce transient ischemia within the tumour vasculature, whereupon the entrapped drug can be released locally. Moreover, tissue permeability is increased due to anoxia, allowing the drug to permeate more easily into the tissue [5].

Albumin microspheres (AMS) have received much attention as possible devices for chemoembolization [6,7]. However, the use of AMS for the release of ADR has some drawbacks. The preparation methods afford only low payloads ( $1-10 \%)$. Furthermore ADR may react with the crosslinking agent during the stabilization process, or may decompose when the microspheres are stabilized at high temperatures [8,9]. AMS are somewhat hydrophobic and surfactants are needed to resuspend them in aqueous solutions. 
Another problem in the use of ADR-loaded AMS is the large burst effect usually observed. In any aqueous medium $80-90 \%$ of the drug is released within $30 \mathrm{~min}$. As a result it is hard to handle the formulation in a clinical situation.

Previously it has been shown that some of these problems do not occur if microspheres are highly negatively charged and thus have ion-exchange properties [10]. With AMS, this has been achieved by the incorporation of carboxylic acid groups containing polyelectrolytes like poly ( $\alpha$ L-glutamic acid) [11] or poly ( $\beta$-L-aspartic acid) [12] into the microspheres. More recently negatively charged, biodegradable vesicles, have been prepared by the incorporation of heparin in albumin microspheres [13,14]. Heparin is a negatively charged mucopolysaccharide. In addition to weak acidic carboxylic acid groups, heparin also contains strong acidic sulphate and sulphamate groups. Heparin and ADR are known to form ionic complexes. Menozzi and Arcamone [15] studied the in vitro interaction of heparin and ADR, whereas Cofrancesco et al. [16] reported that ADR inhibits the anticoagulant activity of heparin in vivo.

Albumin-heparin conjugate microspheres are prepared according to well documented emulsion stabilization techniques used in AMS preparation [6]. The conjugate used in the microsphere preparation is synthesized as described by Hennink et al. [17]. Details on the manufacturing of the conjugates and the microspheres are described elsewhere [18]. Due to the ion-exchange properties of the carrier, ADR loading of the microspheres can be carried out after microsphere preparation by incubating the microspheres in an ADR solution [13]. It has also been demonstrated that AHCMS can be loaded with other drugs of higher molecular weight by a swelling-deswelling process [19].

In this paper the ADR loading and in vitro release characteristics of AHCMS are described. The released material was characterized by HPLC and the activity was tested using a B16 colony formation assay. Furthermore the drug release profile was modelled using ion-exchange and diffusion controlled release kinetics.

\section{Materials and methods}

\section{Materials}

Highly refined olive oil and porcine serum albumin (fraction V) were obtained from Sigma Chemical Company, St. Louis, USA. Glutaraldehyde ( $25 \%$ aqueous solution), obtained from Merck, Darmstadt, Germany, was purified by distillation [20] and adjusted to 2.5, 5 and 25\% w/v aqueous solutions prior to use. Phosphatebuffered saline (PBS) was obtained from NPBI, Emmer Compascuum, The Netherlands. Adriamycin was a kind gift from Farmitalia Carlo Erba, Milan, Italy. A batch of $0.26 \mathrm{mg}$ of ${ }^{14} \mathrm{C}$ adriamycin with a specific activity of $95 \mu \mathrm{Ci} / \mathrm{mg}$, was obtained from Amersham, UK (batch no. 14). It was mixed with $100 \mathrm{mg}$ of unlabelled adriamycin. Soluene ${ }^{\circledR}$ and the scintillation fluids Optifluor $^{\circledR}$, UltimaGold ${ }^{\circledR}$ and Dimilume-30 ${ }^{\circledR}$ were obtained from Packard Instrument B.V., Chemical Operations, Groningen, The Netherlands. All other reagents were obtained, in the highest possible grade, from Merck, Darmstadt, Germany.

\section{Albumin-heparin conjugates}

The preparation and characterization of albumin-heparin conjugates was carried out as described by Cremers et al. [18]. Two different conjugates were prepared from human serum albumin and heparin. An unfractionated conjugate with a heparin content of $10.7 \pm 0.9 \% \mathrm{w} / \mathrm{w}$ (AHC). And a high-affinity conjugate (ha-AHC) prepared by fractionation of an unfractionated conjugate by ion-exchange chromatography using a DEAE-sepharose column. The heparin content of the ha-AHC was $10.2 \pm 1.2 \%$. Furthermore albumin-hcparin conjugates, prepared from porcine albumin with heparin contents of $5.9 \%$ (p-AHC1), $16.0 \%$ (p-AHC2) and $21.5 \%$ (p$\mathrm{AHC} 3$ ) as indicated by the manufacturer, were obtained from Holland Biomaterials Group (HBG), Enschede, The Netherlands. 
TABLE 1

Microspheres used for drug loading and release experiments

\begin{tabular}{lllll}
\hline Code $^{\mathrm{a}}$ & $\begin{array}{l}\text { Glutaraldehyde } \\
\text { concentration } \\
(\%)\end{array}$ & $\begin{array}{l}\text { Reduction } \\
\text { with } \\
\mathrm{NaBH}_{3} \mathrm{CN}\end{array}$ & $\begin{array}{l}\text { Quenching } \\
\text { with } \\
\text { glycine }\end{array}$ & $\begin{array}{l}\text { Heparin } \\
\text { content } \\
(\%)\end{array}$ \\
\hline AHCMS10 & 1.0 & - & - & 10.7 \\
AHCMS10Q $^{\mathrm{b}}$ & 1.0 & - & + & 10.7 \\
AHCMS10RQ $^{\mathrm{b}}$ & 1.0 & + & - & 10.7 \\
AHCMS05 & 0.5 & - & - & 10.7 \\
AHCMS50 & 5.0 & - & - & 10.7 \\
ha-AHCMS10 & 1.0 & - & - & 10.2 \\
PSAMS05 & 0.5 & - & - & 0.0 \\
p-AHC1MS05 & 0.5 & - & - & 5.9 \\
p-AHC2MS05 & 0.5 & - & & 16.0 \\
p-AHC3MS05 $^{\mathrm{d}}$ & 0.5 & & & 21.5 \\
\hline
\end{tabular}

"Numbers refer to crosslink density.

${ }^{\circ}$ Capitals refer to extra process conditoin: $R$, reduction; $Q$, quenching.

'Prepared from conjugate with high-affinity towards DEAE-sepharose ion-exchange column (ha-AHC).

${ }^{d}$ Prepared from porcine-albumin-heparin conjugates with varying heparin content: $\mathrm{p}-\mathrm{AHC} 1,5.9 \%$ heparin; $\mathrm{p}-\mathrm{AHC} 2,16 \%$ heparin; p-AHC3, 21.5\% heparin.

\section{Microsphere preparation}

AHCMS were prepared according to methods developed for the preparation of AMS. In the microsphere preparation, the type of conjugate, i.e., heparin content, the crosslink density, reduction with $\mathrm{NaBH}_{3} \mathrm{CN}$ and quenching with glycine were varied as described previously [18]. In Table 1 the types of microspheres that have been used in drug loading and release experiments are listed.

\section{Adriamycin loading}

In a typical experiment, $10 \mathrm{mg}$ of AHCMS was ultrasonically suspended in $1 \mathrm{ml}$ of a ${ }^{14} \mathrm{C}$-adriamycin solution $(5 \mathrm{mg} / \mathrm{ml}$ water $)$. This suspension was gently rotated for $20 \mathrm{~h}$ at $4^{\circ} \mathrm{C}$, in the dark. The suspensions were then centrifuged, the loading solution was decanted and $1 \mathrm{ml}$ of distilled water was added to rinse the microspheres. After centrifugation, the washing solution was decanted again. This procedure was repeated three more times and was subsequently repeated four times with $1 \mathrm{ml}$ of acetone to dehydrate the microspheres. The microspheres were left overnight at room temperature in the dark to evapo- rate the acetone, and subsequently dried in vacuo for $3 \mathrm{~h}$ at room temperature.

The loading kinetics were determined by monitoring the ${ }^{14} \mathrm{C}$-adriamycin level in solutions during the drug loading. Samples were taken at various time intervals. The maximal payload of the microspheres was determined using ADR loading solutions of increasing concentration (0.5$10 \mathrm{mg} / \mathrm{ml}$ ). ADR concentrations of loading and washing solutions were determined using HPLC. The HPLC system consisted of an ISCO HPLCpump (model 2350), a Rheodyne injection valve (model 7120) equipped with a fixed sample loop of $10 \mu \mathrm{l}$ and a Chrompack C18 microsphere (3 $\mu \mathrm{m})$ column $(100 \times 4.6 \mathrm{~mm}$ i.d. $) . A D R$ and ADR metabolites were detected using fluorimetry by a Perkin Elmer LS4 fluorescence spectrometer at $\lambda_{\mathrm{exc}}=488 \mathrm{~nm}$ and $\lambda_{\mathrm{em}}=588 \mathrm{~nm}$. The mobile phase consisted of $73 \% \mathrm{v} / \mathrm{v}$ acetonitrile, $27 \% \mathrm{v} / \mathrm{v} 0.05 \mathrm{M} \mathrm{KH}_{2} \mathrm{PO}_{4}$, with the final pH adjusted to 4.0 using orthophosphoric acid. A flow rate of $0.5 \mathrm{ml} / \mathrm{min}$ was applied.

\section{Adriamycin payload determination}

\section{Depletion/recovery method}

The amount of ADR remaining in the loading solution and the ADR amounts in the washing 
solutions were determined by ${ }^{14} \mathrm{C}$ scintillation counting or by UV/Vis measurements. The payload was calculated from the adriamycin depletion of the loading solution and the adriamycin recovered from the washing and dehydration solutions. To have an indication of the stability of the ADR during these procedures, samples of the ADR in the washing and dehydration solutions were analysed by HPLC.

\section{Degradation method}

ADR-loaded microspheres $(1 \mathrm{mg}$ ) were degraded in $1 \mathrm{ml}$ of Soluene ${ }^{\circledR}$ for $65 \mathrm{~h}$ at $37^{\circ} \mathrm{C}$, and subsequently for 5 more hours at $65^{\circ} \mathrm{C}$. After cooling down to room temperature, $15 \mathrm{ml}$ of Dimilume- $30^{\circledR}$ was added, and the mixture was vortexed. Radioactivity was determined using a scintillation counter (Hewlett Packard 1900 CA Tri-Carb Liquid Scintillation Analyzer, USA). As a control, $100 \mu \mathrm{l}$ of a ${ }^{14} \mathrm{C}$-adriamycin solution $(0.5 \mathrm{mg} / \mathrm{ml})$ was pipetted into a vial containing approximately $1 \mathrm{mg}$ of empty microspheres. The controls were further treated in the same way as the other vials. The payload of the microspheres was calculated using the specific activity that was determined from the controls.

\section{Adriamycin release}

Release experiments were carried out using a batch system. Microspheres ( $\pm 1 \mathrm{mg}, 5-35 \mu \mathrm{m}$ ) were weighed on an aluminium foil basket, which was put into a $20 \mathrm{ml}$ polypropylene (PP) vial. Two hundred $\mu$ l ethanol was added for better wetting and the microspheres were ultrasonically resuspended. No drug release was observed during this procedure. At $t=0,20 \mathrm{ml}$ of release medium (PBS; human plasma, containing $0.02 \%$ sodium azide; $5 \%$ glucose solution; $5 \%$ albumin solution or distilled water) was added and the counting vials were put into a shaking bath at $37^{\circ} \mathrm{C}$. The amplitude of the shaking was $2.5 \mathrm{~cm}$ and the frequency, approximately $2 \mathrm{~Hz}$. At intervals, the PP vials were taken from the bath and shaken vigorously to homogenize the suspension. A $0.5 \mathrm{ml}$ sample was pipetted from the vial into a $1.5 \mathrm{ml}$ Eppendorf ${ }^{\circledR}$ cup. After centrifugation for $5 \mathrm{~s}$ at $13000 \mathrm{rpm}$, two $200-\mu 1$ samples were taken from the supernatant, and transferred into 6-ml counting vials. Scintillation medium (Optifluor ${ }^{\circledR}$ or UltimaGold ${ }^{\circledR}$ ) was added $(4 \mathrm{ml})$ and the activity was determined. The release profile was calculated from the activity of the samples.

In order to mimic a clinical situation, release experiments $(n=4)$ were carried out in which the microspheres ( $\pm 1 \mathrm{mg}$ ) were resuspended in 10 $\mathrm{ml}$ of injection medium $(5 \% \mathrm{w} / \mathrm{v}$ glucose solution ) at room temperature. The vials were left to stand in the dark. Every $15 \mathrm{~min}$, they were shaken gently. After $1.5 \mathrm{~h}, 10 \mathrm{ml}$ of a warm $\mathrm{NaCl}$ solution was added. In this way the temperature of the medium was instantaneously increased to $37^{\circ} \mathrm{C}$ and the $\mathrm{NaCl}$ concentration was increased to a physiological level. The vials were placed in the shaking bath at $37^{\circ} \mathrm{C}$. The release was monitored at 30 -min time intervals as described previously.

The influence of the ADR release on the swelling of the microspheres was investigated by measuring the release of $\mathrm{ADR}$ and the size distribution of the AHCMS simultaneously. ADRloaded AHCMS05 (16 mg) were resuspended in $150 \mu \mathrm{l}$ ethanol. At $t=0$ the suspension was added to $100 \mathrm{ml}$ of PBS in the sample chamber of a Horiba LA 500 laser diffraction particle size distribution analyser (Horiba, Kyoto, Japan). The suspension was continuously agitated and circulated through the measuring chamber. After various time intervals, two $1-\mathrm{ml}$ samples were taken from the suspension and centrifuged at 13000 rpm. From the supernatant $0.9 \mathrm{ml}$ was pipetted into a 1.5-ml Eppendorf ${ }^{\circledR}$ cup and stored until ADR determination. This was done by UV/Vis spectroscopy. $\mathrm{E}_{494}$ was measured with an Uvikon 930 spectrophotometer (Kontron Instruments, Rotkreuz, Switzerland). The size distribution of the microspheres was also determined (between 0.5 and $200 \mu \mathrm{m}$, in 40 channels of 5 $\mu \mathrm{m}$ each ) at several time intervals.

\section{B16 colony formation assay of released adriamycin}

A standard suspension of $100 \mathrm{~B} 16$ cells $/ 3 \mathrm{ml}$ was prepared in Dulbecco's Modified Eagle's 
Medium, containing 10\% new-born calf serum (DMEM). Three $\mathrm{ml}$ of this suspension was added to wells of a six-well tissue culture plate. Cells were left to adhere for $24 \mathrm{~h}$ after which a $15-\mu \mathrm{g} / \mathrm{ml}$ ADR solution was added to a final concentration of $3,1,0.3,0.1,0.03,0.01$ or $0 \mu \mathrm{g} /$ ml ADR ( $n=6$ per concentration point, $n=10$ in controls). At the highest concentration ( $3 \mu \mathrm{g} /$ $\mathrm{ml}$ ), $0.6 \mathrm{ml}$ of medium was removed prior to the addition of the ADR solution. After $30 \mathrm{~min}$ exposure the ADR containing medium was removed, the wells were washed with PBS and fresh medium was added. The wells were kept for 5 days at $37^{\circ} \mathrm{C}\left(5 \% \mathrm{CO}_{2}\right)$. The medium was then removed and the wells were washed with PBS. Cells and colonies were fixed using ethanol and stained with crystal violet. Colonies ( $\geq 50$ cells) were counted using a light microscope. These experiments were carried out with four types of ADR: ADR as obtained from Farmitalia, ${ }^{14} \mathrm{C}$ $A D R$ as used in the drug loading and release experiments, ${ }^{14} \mathrm{C}$-ADR as released from AHCMS10 and ${ }^{14} \mathrm{C}$-ADR as released from AHCMSIORQ (after $2 \mathrm{~h}$ release in PBS). The results from this experiment were analysed by probit-analysis.

\section{Results and Discussion}

Albumin-heparin conjugate microspheres were prepared from a prefabricated albumin-heparin conjugate by an emulsion-stabilization technique. Since endogenous materials are used it is expected that the microspheres will be biocompatible and biodegradable. Due to the introduction of strong anionic groups by heparin incorporation, the microspheres exert ion-exchange properties. The biodegradable ion-exchange microspheres were investigated as possible adriamycin delivery devices to be used in intra-arterial chemo-embolization studies for targeting to the liver.

Albumin-heparin conjugates were prepared as described previously [ 17,18$]$. The ADR binding and release properties of the microspheres are predominantly determined by the ionic interaction between the drug and the heparin moieties of the conjugate. Increasing heparin contents will thus result in higher payloads. DEAE ion-exchange chromatography enables fractionation of the conjugate, yielding ha-AHC with a higher charge-density as compared to unfractionated AHC. This will also result in increased payloads.

Microspheres with a diameter of 5-35 $\mu \mathrm{m}$ were prepared by the emulsion stabilization method. Crosslink density, reduction and quenching did not change the size distribution [18]. Using the high-affinity conjugate, a more viscous aqueous solution was obtained (at the same conjugate concentration), resulting in larger microspheres with a larger size distribution. This is in accordance with the results obtained with AMS. The diameter of AMS increases with increasing viscosity of the aqueous phase as well as with decreasing stirring speed, decreasing viscosity of the organic phase and increasing amount of aqueous phase $[6,18]$.

Compared with AMS, the AHCMS are more hydrophilic and relatively easy to resuspend in aqueous medium. This enables resuspension of AHCMS in the ADR containing loading solution. Due to the negatively charged heparin moieties in the microspheres, the loading of the positively charged ADR is a fast and efficient process as can be seen in Fig. 1. If an ADR/ AHCMS ratio of $1 / 2(w / w)$ was used, drug loading was complete after $2-3 \mathrm{~h}$ and approximately $90 \%$ of the drug initially present in the

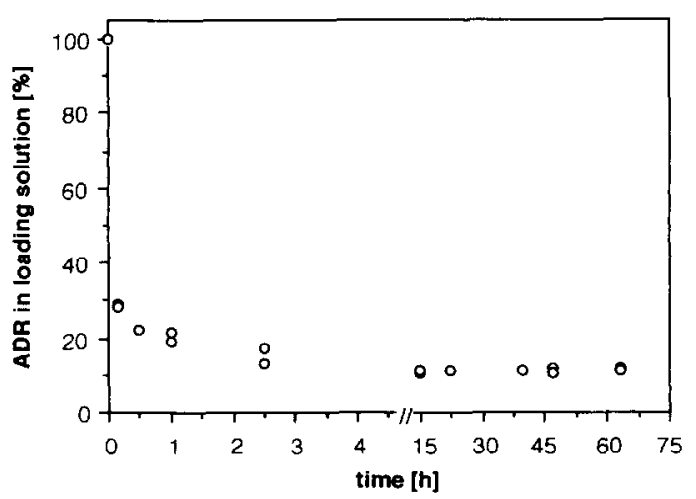

Fig. 1. Relative amount of ADR in the loading solution during ADR loading of $10 \mathrm{mg}$ of AHCMSO5 using $1 \mathrm{ml}$ of an ADR solution of $5 \mathrm{mg} / \mathrm{ml}$; ADR $/$ AHCMS ratio $=0.5 \mathrm{mg} / \mathrm{mg}$ $(n=4)$. 
loading solution was entrapped in the microspheres.

The binding capacity of ADR to AHCMS was limited. At higher concentrations the ADR binding became saturated. Increasing the concentration in the loading solution to an ADR/AHCMS ratio of $1 / 1(\mathrm{w} / \mathrm{w})$ gave only a small increase in payload. This is demonstrated in Figs. 2 and 3 in which the maximum ADR payload of the micro-

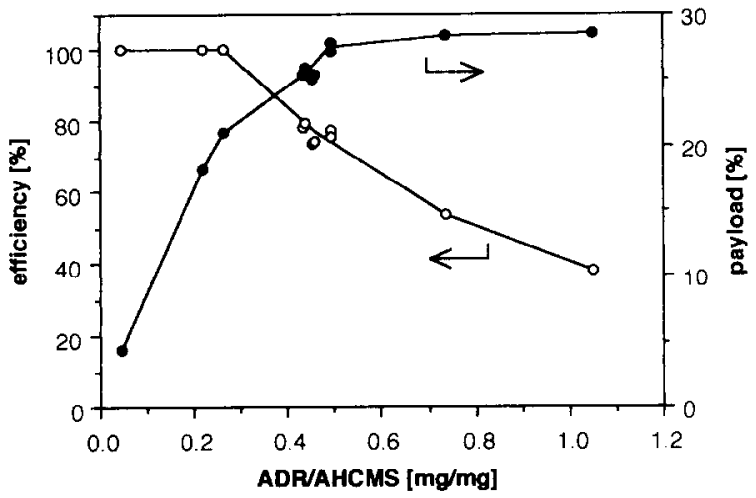

Fig. 2. Influence of the ADR/AHCMS ratio on the payload $(-)$ and the loading efficiency (O), after $24 \mathrm{~h}$ of drug loading $(n=2)$. AHCMS $(10 \mathrm{mg})$ were suspended in $1 \mathrm{ml}$ of ADR solution $(0.5-10 \mathrm{mg} / \mathrm{ml})$. The payloads were determined using the depletion method.

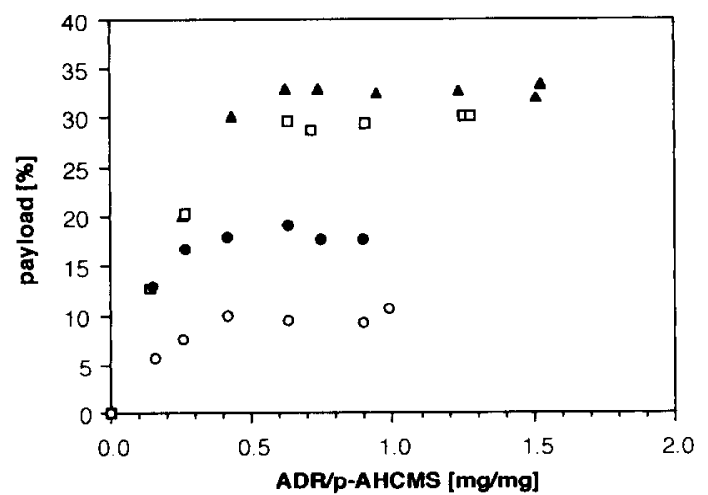

Fig. 3. Influence of the ADR/p-AHCMS ratio on the payload after $24 \mathrm{~h}$ of drug loading $(n=4)$. p-AHCMS ( $5 \mathrm{mg}$ ) were suspended in $1 \mathrm{ml}$ of ADR solution $(0.8-8.0 \mathrm{mg} / \mathrm{ml}) . O$, PSAMS ( $0 \%$ heparin);, p-AHC1MS ( $5.9 \%$ heparin); $\mathbf{\square}$, p-AHC2MS ( $16.0 \%$ heparin); $\Delta$, p-AHC3MS (21.5\% heparin). The payloads were determined using the depletion method. spheres as a function of the concentration of the loading solution is given.

Fig. 2 also shows that the efficiency of the ADR loading is influenced by the ADR/AHCMS ratio used in the loading procedure. The loading efficiency, expressed as the relative ADR depletion from the loading solution, was almost $100 \%$ up to the level at which the maximal loading capacity was reached. This also indicates that under these circumstances, no unbound ADR is present. Unbound ADR, removed during the washing and dehydration procedure would give a lower efficiency.

The payloads obtained with the different types

TABLE 2

Adramycin payloads of AFCMS

\begin{tabular}{lll}
\hline MS-type $^{\mathrm{a}}$ & \multicolumn{2}{l}{ Payload } \\
\cline { 2 - 3 } & Depletion ${ }^{14} \mathrm{C}^{\mathrm{b}}$ & $\begin{array}{l}\text { Degradation } \\
{ }^{14} \mathrm{C}^{\mathrm{c}}\end{array}$ \\
\hline AHCMS10 & $22.8 \pm 2.2$ & $25.6 \pm 1.3$ \\
AHCMS10Q & $24.4 \pm 1.6$ & $29.0 \pm 1.8$ \\
AHCMS10RQ & $22.6+0.5$ & $26.5 \pm 1.0$ \\
AHCMS50 & $29.5 \pm 1.0$ & $27.9 \pm 1.7$ \\
ha-AHCMS10 & $32.2 \pm 0.8$ & $31.9 \pm 2.4$ \\
\hline
\end{tabular}

Microsphere code are explained in Table 1.

${ }^{b}$ Calculated from the results obtained by the ADR depletion/ recovery method $(n=4)$.

cCalculated from the results obtained by the degradation method $(n=2)$.

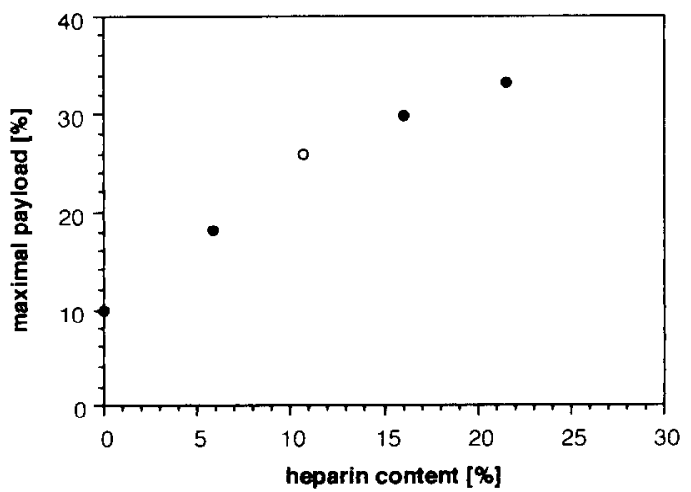

Fig. 4. Maximal ADR payloads as a function of the heparin contents of albumin-heparin conjugate microspheres: 1 , MS prepared from PSA or p-AHC; $\bigcirc$, AHCMS05. 
of microspheres are given in Table 2. Figs. 3 and 4 show the influence of the heparin content of the microspheres on the drug loading capacity of p-AHCMS. With increasing heparin content, higher payloads can be reached. Fig. 4 also shows that p-AMS can bind some ADR, although the payload is less than that of p-AHCMS.

From Table 2 and Fig. 4 it can be concluded that the charge density of the heparin and the heparin content are the most important parameters determining the payload. Previously, Menozzi and Arcamone [15] have determined the ADR-binding capacity of heparin. They found that $3.3 \mathrm{~mol}$ of ADR could be bound per mol of hexosamine residues of heparin. It was determined that, in solution, the unfractionated albumin-heparin conjugate had an ADR binding capacity of $3.2 \mathrm{~mol}$ of ADR $/ \mathrm{mol}$ of hexosamine residues (data not shown). Therefore the ADRheparin binding appears not to be influenced by the covalent coupling of albumin and heparin.

The ADR-heparin binding experiments with solutions suggest that several types of drug binding occur. Next to an initial electrostatic binding between the basic group of the aminosugar moiety of the drug and the polyanionic macromolecule, the drug binding may be enhanced by self-association of ADR due to hydrophobic interactions between ADR molecules [15]. Formation of ADR aggregates in concentrated solutions is a well know phenomenon, which was described previously [21].

As pointed out before, fractionation of the conjugate based on ionic interaction with DEAE sepharose, yields a conjugate which will also show more affinity towards ADR as compared to unfractionated AHC. Accordingly the ADR payloads of microspheres prepared from the high-affinity conjugate (ha-AHCMS10) were higher than that of microspheres prepared from unfractionated conjugate (32\% instead of approximately $27 \%$, Table 2 ). The importance of the heparin and the heparin-ADR interaction is also demonstrated by the increased ADR binding capacity with increasing heparin content (Fig. 3). Furthermore, Table 2 shows that the concentration of the crosslinking agent, quenching and re- duction had virtually no influence on the payload. From these results it can be concluded that the charge density of the matrix material is the most important factor determining the final payload. The drug loading process is a very attractive one since it allows effective drug loading with little or no drug loss during the procedure.

The payloads determined by the depletion/recovery method or by the degradation method are in good agreement. This shows that the more convenient, widely used depletion/recovery method is reliable. A disadvantage of the two methods is that the quality of the incorporated adriamycin cannot be monitored. However, HPLC analysis of the material recovered in the washing steps (data not shown) showed that no degradation of the adriamycin took place during drug loading and washing steps.

ADR release from AHCMS is controlled by the ionic strength of the medium, as is shown in Fig. 5. The release in ion-containing media such as phosphate-buffered saline and human plasma was very fast. Almost all of the drug was released within $2 \mathrm{~h}$. In non-ionic media, however, only $30 \%$ of the drug was released. The ion controlled release provides a method to prepare an ADRAHCMS suspension containing little or no free drug. This is demonstrated in Fig. 6. Maximally $30 \%$ of the drug is released prior to 'injection'.

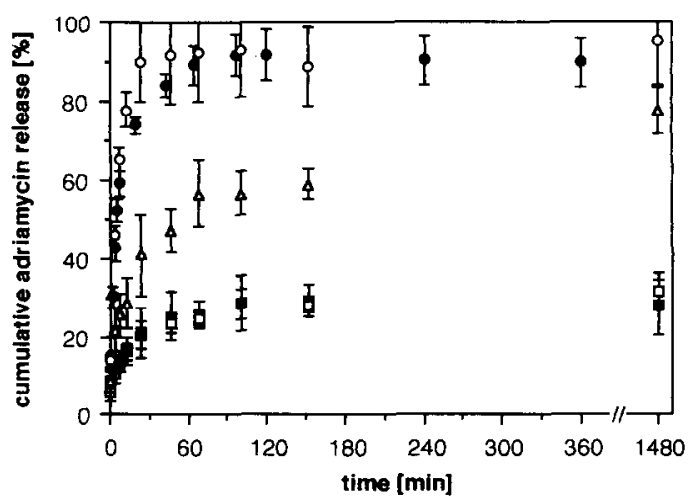

Fig. 5. Cumulative ADR release from AHCMSO5 in several release media at $37^{\circ} \mathrm{C}$. - phosphate-buffered saline; $O$, human plasma containing $0.02 \%$ sodium azide; $\Delta, 5 \% \mathrm{w} / \mathrm{v}$ albumin solution; $\square$, distilled water; $\square, 5 \% \mathrm{w} / \mathrm{v}$ glucose solution. Error bars indicate $\mathrm{SD}(n=4)$. 


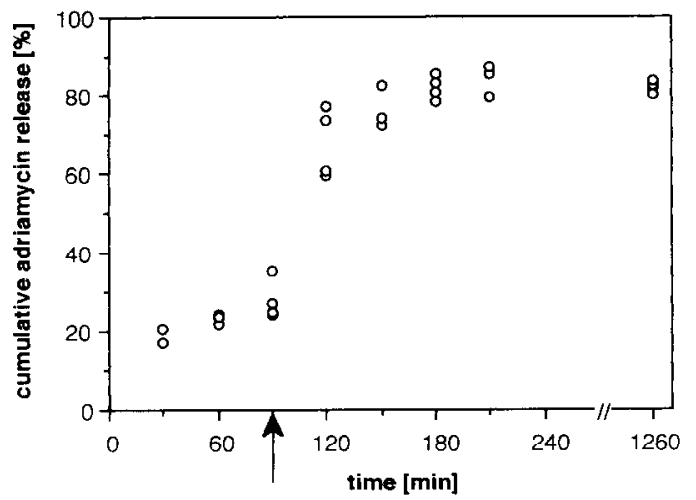

Fig. 6. Cumulative ADR release from AHCMS10 in $5 \% \mathrm{w} / \mathrm{v}$ glucose solution at $20^{\circ} \mathrm{C}$. At $t=90 \mathrm{~min}$ (arrow), warm saline was added to increase the $\mathrm{NaCl}$ concentration of the release medium to a physiological level and the temperature to $37^{\circ} \mathrm{C}$ $(n=4)$.

Upon increasing the salt concentration up to physiological levels by addition of a salt solution, thus imitating injection, the remaining ADR was released immediately. Another important feature is that ADR remaining in the AHCMS after the initial release is not released in fresh glucose solution, indicating that the ADR released prior to the injection can be removed using a simple washing step with non-ionic medium. This would be impossible with AMS, releasing the ADR in any aqueous solution, regardless of the ionic strength. Comparing AHCMS and AMS the major improvement of AHCMS is the obtained control over the onset of the drug release.

The drug release in non-ionic medium (30\%) was unexpected since the ADR ought to be bound ionically to the matrix. Moreover, no drug was released in water during the washing steps in the drug loading procedure. It was already mentioned, however, that the electrostatic interaction of the ADR and the matrix is not the only mode of binding in this system. Apart from this type of binding, a substantial amount of ADR will be bound by self-association of ADR. It is suggested that due to the dehydration steps with acetone during the drug loading procedure, some of the hydrophobically bound ADR is detached from the ionically bound ADR. Previously, it has been shown that the dimerization equilibrium of anthracyclines such as adriamycin and daunomycin is shifted towards free drug in the presence of organic solvents [22,23]. This drug remains trapped in the dehydrated microsphere as unbound drug. Upon swelling in water, this drug is released, regardless of the ionic strength of the medium.

Fig. 7 shows the ADR release profiles from several types of AHCMS. As can be seen from this figure, the ADR release profile was not influenced by the quenching and reduction steps, nor by fractionation of the conjugate. All microspheres crosslinked with $1.0 \%$ glutaraldehyde released the drug within $45 \mathrm{~min}$. There was an influence of crosslink density, i.e., the drug release from microspheres crosslinked with $5.0 \%$ glutaraldehyde (AHCMS50) was slower. The haAHCMS50 seemed to release the drug even slower. The ha-AHCMS50, however, have been prepared under different emulsification conditions, resulting in larger microspheres (12-80 $\mu \mathrm{m}$ instead of $5-35 \mu \mathrm{m}$ ).

Fig. 7 also shows that not all of the drug was released from the microspheres. In the best case (AHCMS10RQ) 90\% was released, most other types of microspheres released approximately $80 \%$ of the ADR. If this is due to covalent binding of ADR to the unreacted aldehyde groups of the glutaraldehyde, quenching should result in

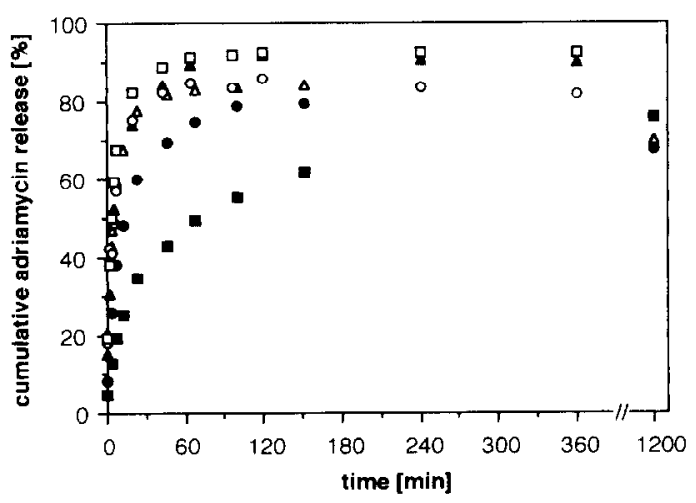

Fig. 7. Cumulative ADR release from several types of AHCMS in phosphate buffered saline at $37^{\circ} \mathrm{C}$. $\boldsymbol{\Delta}$, AHCMS10; $\mathrm{O}$, AHCMS10Q; $\square$, AHCMS10RQ; AHCMS50; $\triangle$, haAHCMS10; $\square$, ha-AHCMS50 $(n=4)$. 
more complete drug release. AHCMS10Q have been quenched by addition of glycine during the microsphere preparation and the maximal release of these microspheres was not different from that of AHCMS50 or ha-AHCMS10. Quenching with glycine in the presence of a reducing agent (AHCMS10RQ), however, did result in relatively higher drug releases. This indicates that adequate quenching may only be achieved if a reducing agent is present. It also indicates that some of the drug remaining in the non-quenched microspheres after drug release, may be covalently bound to the matrix. Since ADR release from AHCMS10RQ is only $90 \%$, covalent coupling of ADR to the albumin-heparin conjugate matrix does not account for all of the drug remaining in the microspheres. An explanation for the drug remaining in the microspheres is the stabilization of electrostatically bound ADR by hydrophobic interactions with other ADR molecules, which was already mentioned before. Menozzi and Arcamone [15] found that in solution, only $50 \%$ of the ADR could be displaced from the heparin by $\mathrm{Na}^{+}$at physiological concentrations. ADR-conjugate binding studies carried out with solutions showed that, in phophate buffered saline, only $80 \%$ of the drug was displaced from the conjugate (data not shown). Based on these results it is likely that some of the ADR aggregates, electrostatically bound to the matrix cannot be detached by counterions in the release medium.

From the HPLC analysis of the released material it was concluded that after $2 \mathrm{~h}$ release at $37^{\circ} \mathrm{C}$ no degradation products of ADR were formed (data not shown). The dose/response curves of the different ADR samples, as calculated from the B16 CFA, are shown in Fig. 8. There were no significant differences between the $\mathrm{LD}_{50}$ values of the four types of ADR used in the tests, as determined by probit-analysis. These results prove that during drug loading and in vitro release procedures no degradation of the ADR occured. The released material was still active ADR.

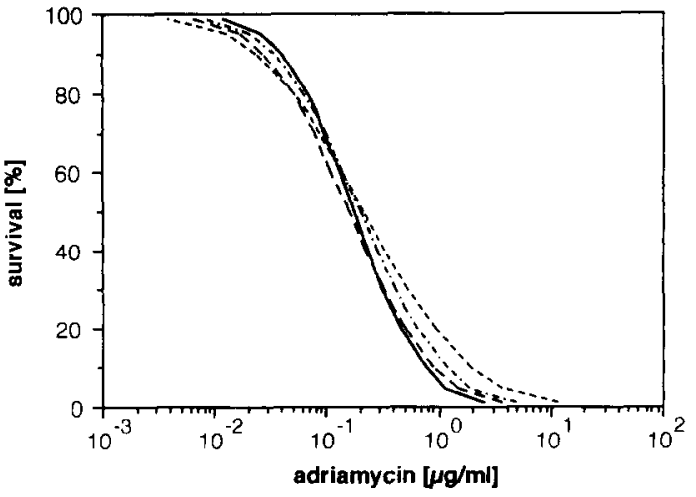

Fig. 8. Dose-response curves for various ADR samples as determined by a $\mathrm{B} 16$ colony formation assay and calculated using probit analysis. —, ADR; -..-, ${ }^{14} \mathrm{C}-\mathrm{ADR} ; . \cdot . \cdot . \cdot . \cdot{ }^{14} \mathrm{C}-$ ADR released from $\mathrm{AHCMS10} ;-----,{ }^{14} \mathrm{C}-\mathrm{ADR}$ released from AHCMS10RQ.

\section{Modelling of adriamycin release}

Considering the ion-exchange properties of the AHCMS several steps in the adriamycin release process can be distinguished:

- swelling of the microspheres in the release medium;

- in-diffusion of the counter ions;

- actual ion-exchange of counter ions and drug;

- out-diffusion of the drug.

As was shown before, the swelling behaviour of the AHCMS is sensitive to the ionic strength of the medium, i.e., to the presence of ions [18]. Possibly, the swelling will change during the drug release, i.e., during the exchange of larger hydrophobic drug molecules with small ions. Fig. 9, however, shows that the swelling is very fast as compared to the drug release. In this figure the $D_{25 \%}, D_{50 \%}$ (median) and $D_{75 \%}$, which are the diameters where the given percentage of particles is smaller than that size, as well as the ADR release profile are presented. The figure also shows that the size distribution of the AHCMS was constant throughout the ADR release.

Assuming that the actual ion-exchange is very fast as compared to the diffusion processes, the drug release rate is mainly controlled by the in- 


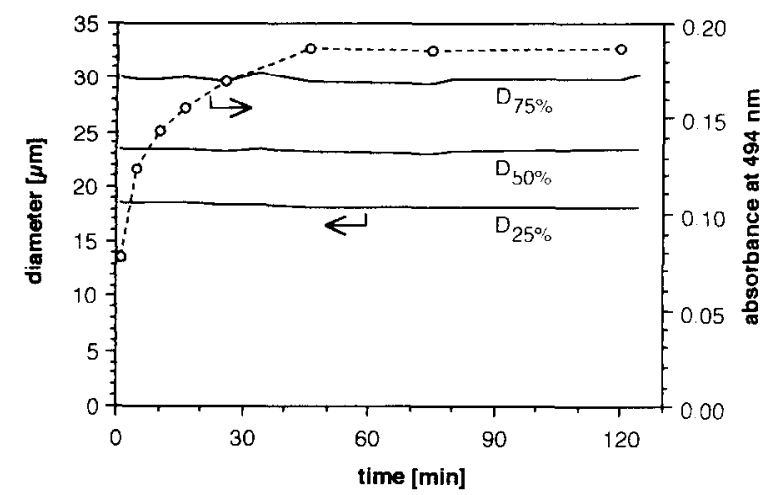

Fig. 9. Size distribution of AHCMS05 during ADR release in phosphate buffered saline at $25^{\circ} \mathrm{C}$. $\cdots 0^{\cdots} \cdots$, ADR release expressed as the $E_{494}$ of the release medium; - - size distribution as indicated by $D_{25 \%}, D_{50 \%}$ and $D_{75 \%}$, which are the diameters where the given percentage of particles is smaller than that size.

diffusion of the counter ions and the out-diffusion of the drug.

Ion-exchange kinetics have been described extensively by Hellferich [24]. Briefly, the total counter ion concentration (ions and drug) must remain constant (in equivalents) and equal to the amount of charged groups in the matrix. This implies that the fluxes (in equivalents) of the counter ions (in-diffusion) and the drug (outdiffusion) should be equal. So, instead of two independent diffusion processes, there is a rigid coupling between the two fluxes and the kinetic behaviour can be calculated from only one diffusion equation.

$J_{\mathrm{A}}=-D_{\text {int }} \operatorname{grad} C_{\mathrm{A}}$

where the interdiffusion coefficient is:

$D_{\text {int }}=D_{\mathrm{A}} D_{\mathrm{B}} \frac{z_{\mathrm{A}}^{2} C_{\mathrm{A}}+z_{\mathrm{B}}^{2} C_{\mathrm{B}}}{z_{\mathrm{A}}^{2} C_{\mathrm{A}} D_{\mathrm{A}}+z_{\mathrm{B}}^{2} C_{\mathrm{B}} D_{\mathrm{B}}}$

Hellferich [24] has given a detailed description of these equations.

Essentially, the release behaviour can be described by a diffusion equation in which the interdiffusion coefficient $\left(D_{\text {int }}\right)$ is determined by the relative concentrations and the diffusion coefficients of the drug and the counter ion (resp. $C_{\mathrm{A}}, C_{\mathrm{B}}, D_{\mathrm{A}}$ and $\left.D_{\mathrm{B}}\right)$. This interdiffusion coefficient is not constant but is predominantly con- trolled by the ion which is in the minority. From Eq. 2 it can be seen that $D_{\text {int }}=D_{\mathrm{A}}$ if $C_{\mathrm{A}} \ll C_{\mathrm{B}}$. In the case of ion-exchange in the microspheres, it may be assumed that after swelling, the drug concentration is much lower than the concentration of the counter ion. So the interdiffusion coefficient will almost equal the drug diffusion coefficient. Hence, the ADR release from AHCMS will be predominantly diffusion controlled.

Mathematical descriptions of diffusion processes, suited to describe drug release from spherical monolithic devices were derived by Crank [25]. For the benefit of simplicity, early and late time approximations of these equations are generally used [26-28]. The general equation derived for spherical geometry by Crank [25] and the early and late time approximations are given in Eqs. 3, 4 and 5, respectively.

$\frac{M_{t}}{M_{\infty}}=1-\frac{6}{\pi^{2}} \sum_{n=1}^{\infty} \frac{1}{n^{2}} \exp \left[\frac{-D n^{2} \pi^{2} \mathrm{t}}{r^{2}}\right]$

Early time approximation:

$\frac{M_{t}}{M_{\infty}}=6\left[\frac{D t}{\pi r^{2}}\right]^{0.5}-3 \frac{D t}{r^{2}}$

Late time approximation:

$\frac{M_{t}}{M_{\infty}}=1-\frac{6}{\pi^{2}} \exp \left[\frac{-D \pi^{2} t}{r^{2}}\right]$

From Eqs. 1 and 2 it was concluded that the ionexchange kinetics could be described by a single diffusion equation in which the diffusion coefficient was replaced by an interdiffusion coefficient. This indicates that the adriamycin release from AHCMS could be described by Eqs. 4 and 5 , in which the diffusion coefficient $D$ is the interdiffusion coefficient. In physiological salt solution, this interdiffusion coefficient will be constant and almost equal the diffusion coefficient of the adriamycin.

Another problem is that the experimental release data are data obtained from a population of microspheres of varying diameter. Donbrow 
et al. [29] showed that in the case of microcapsules, the zero-order single particle release profiles may add up to first order population release kinetics. The effect of the size distribution of ionexchange monolithic AHCMS on the release was investigated. For this purpose the size distribution of the microspheres, presented in Fig. 10, was determined during the drug release.

Fig. 11 shows the results of the ADR release from AHCMS05 in PBS at $37^{\circ} \mathrm{C}$ together with the release profile calculated by fitting Eq. 6 to these data, with $f_{\mathrm{i}}$ being the fraction of particles with a diameter of $r_{\mathrm{i}}$ (as represented in Fig. 10).

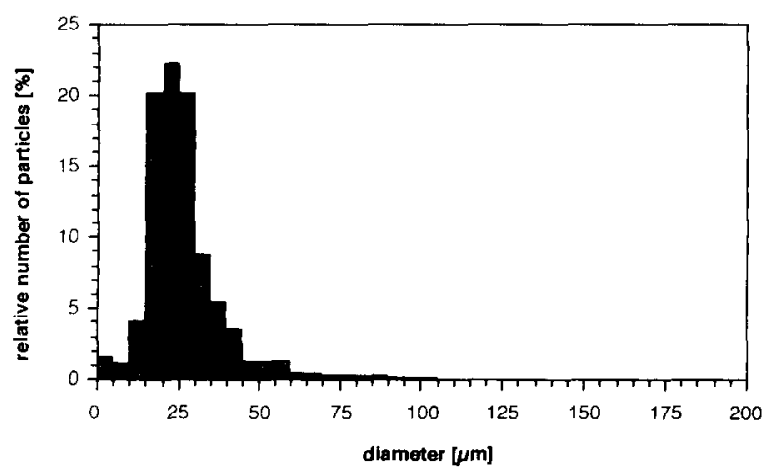

Fig. 10. Size distribution of AHCMSO5 during ADR drug release in PBS at $25^{\circ} \mathrm{C}$, determined in 40 channels of $5 \mu \mathrm{m}$ each.

Mean diameter, $30.8 \mu \mathrm{m}$; median diameter, $23.7 \mu \mathrm{m}$.

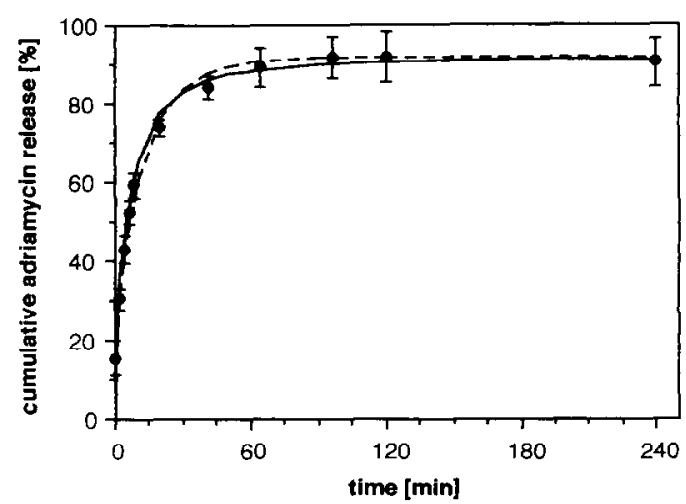

Fig. 11. Cumulative ADR release from AHCMS05 in PBS. - experimental data, error bars indicate $\mathrm{SD}$; - , release profile calculated with Eq. 6 to fit the experimental data using the size distribution presented in Fig. 10; ----, release profile calculated with Eq. 6 with $f_{i}=1$ and $r_{i}=30.8$ (mean diameter), using the $D_{\text {int }}$ from previous fit.
From this fit the interdiffusion coefficient was determined to be $2.5 \mathrm{E}-10 \mathrm{~cm}^{2} \mathrm{~s}^{-1}$. Fig. 11 also shows the release profile calculated with this interdiffusion coefficient and the mean diameter $(30.8 \mu \mathrm{m})$ of the population. This mean-diameter profile was calculated using Eq. 6 with only one $f_{\mathrm{i}}$ and $r_{\mathrm{i}}$ being 1 and $30.8 \mu \mathrm{m}$, respectively. Calculating the mean-diameter release profile using Eqs. 4 and 5 did not alter the result.

$\frac{M_{t}}{M_{\infty}}=\sum_{l i=1}^{40} f_{i}\left\langle 1-\frac{6}{\pi^{2}} \sum_{n=1}^{20} \cdot \frac{1}{n^{2}} \exp \left[\frac{-D n^{2} \pi^{2} t}{r_{i}^{2}}\right]\right\rangle$

In Fig. 11 the experimental release data as well as the 'population'-fit and the calculated 'meandiameter' release profile are presented. It is obvious that, although the profiles are not exactly the same, the mean-diameter profile fits the experimental data as well as the population release kinetics. This indicates that within the particle size distributions used in these release experiments, a 'mean diameter'-fit procedure using Eqs. 4 and 5 , which is carried out more easily, is allowed.

Fig. 12 shows the release profiles of adriamycin from AHCMS10 in various media, fitted using the early and late time approximations of the diffusion controlled release model (Eqs. 4 and

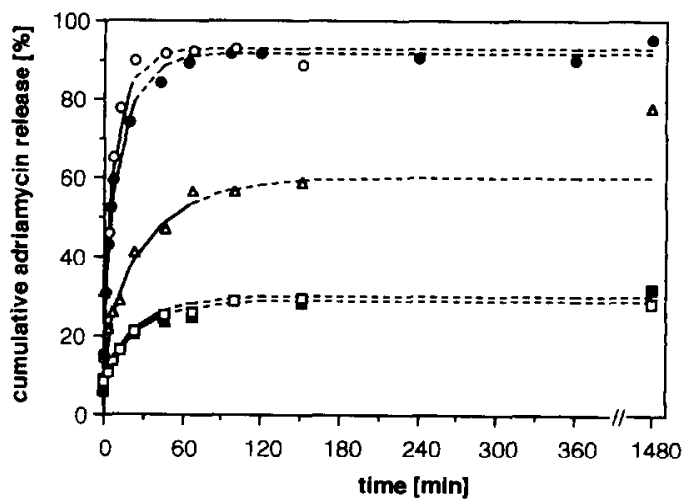

Fig. 12. ADR release from AHCMS10 in several release media at $37^{\circ} \mathrm{C}$, fitted with early $(-)$ and late (----) time approximations of diffusion controlled drug release kinetics from spherical monolithic devices (Eqs. 4 and 5). phate buffered saline; $O$, human plasma containing $0.02 \%$ sodium azide; $\triangle, 5 \% \mathrm{w} / \mathrm{v}$ albumin solution; $\square$, distilled water; $\square, 5 \% \mathrm{w} / \mathrm{v}$ glucose solution. 
$5)$. The release profiles can be fitted very well with the early and late time approximations of the Fickian diffusion controlled release kinetics. In ionic medium such as physiological salt solution and human plasma, the diffusion coefficient is the $D_{\text {int }}$ of the ion-exchange kinetics. In nonionic medium such as distilled water or $5 \% \mathrm{w} / \mathrm{v}$ glucose solution, the diffusion coefficient is simply the diffusion coefficient of unbound ADR.

In the case of $5 \% \mathrm{w} / \mathrm{v}$ albumin solutions, the situation is more complicated. In drug loading studies of AHCMS, Kwon et al. [19] showed that FITC-dextrans with an average weight molecular weight of 17200 could only be loaded up to a payload of $0.5 \%$ after $50 \mathrm{~h}$ of incubation. Therefore it is not likely that albumin with a molecular weight of approximately 66000 will diffuse into the microspheres. Elemental analysis showed that the albumin contained $0.5 \%(\mathrm{w} / \mathrm{w})$ of sodium. Thesc sodium ions account for the ion-exchange that takes place during the ADR release in the albumin solution. The sodium, however, will also be bound to the albumin and the concentration in the microsphere will thus be relatively low and will change during drug release. As a result of this, the $D_{\text {int }}$ will neither be constant nor almost equal to the diffusion coefficient of the ADR. Hence, straightforward fitting of the release profiles using Eqs. 4 and 5 is not adequate anymore.

\section{Conclusions}

Albumin-heparin conjugate microspheres are biodegradable ion-exchange particles prepared from endogenous materials. The AHCMS could easily be loaded with the positively charged adriamycin up to payloads of $10-34 \%$, depending on the type of conjugate used. Drug release was ionically controlled and this enabled us to prepare injectable ADR-AHCMS suspensions in which the drug release was delayed until contact with body fluids. It was also shown that the drug release can be described by combining well documented ion-exchange kinetics and diffusion models.

\section{Acknowledgements}

The authors wish to acknowledge The Netherlands Organization for Scientific Research (NWO) and Theratech Inc. Salt Lake City, Utah, for financially supporting this research and Farmitalia Carlo Erba for the kind gift of adriamycin. Thanks are also due to Jolanda Melching of the University of Twente, to Marika Hemmen of the Hogeschool Drenthe and to Ingrid Smolders of The Netherlands Cancer Institute for their contributions to the work described in this paper.

\section{References}

1 S.S. Bielack, R. Erttmann, K. Winkler and G. Landbeck, Doxorubicin: effect of different dosing schedules on toxicity and anti-tumour efficacy, Eur. J. Cancer Clin. Oncol., 5 (1989) 873-882.

2 J.H. Doroshow, Doxorubicin-induced cardiac toxicity, New Engl. J. Med., 324 (1991), 843-845.

3 E. Tomlinson, Microsphere delivery systems for drug targeting and controlled release, Int. J. Pharm. Tech. Prod. Mfr., 4 (1983) 49-57.

4 S.S. Davis and L. Illum, Polymeric microspheres as drug carriers. Biomaterials, 9 (1988) 111-115.

5 H.P.J.M. Noteborn and J.G. McVie, Chemoembolization in regional chemotherapy. In L. Domellöf (Ed.), Drug delivery in Cancer Treatment, ESO monographs, Springer Verlag, Berlin-New York, 1989, pp. 55-78.

6 J.J. Burger, E. Tomlinson, E.M.A. Mulder and J.G. McVie, Albumin microspheres for intra-arterial tumour targeting. I. Pharmaceutical aspects, Int. J. Pharm., 23 (1985) 333-344.

7 C.S. McArdle, H. Lewi, D. Hansell, D.J. Kerr, J. McKillop and N. Willmott, Cytotoxic-loaded albumin microspheres: a novel approach to regional chemotherapy, Br. J. Surg., 75 (1988) 132-134.

8 K.J. Widder, P.A. Marino, R.M. Morris and A.E. Senyei, Targeting antineoplastic agents using magnetic albumin microspheres, Polymers Biol. Med., 2 (1983) 201-230.

9 S.L. Law, C.H. Chiang, F.M. Lin and G.W. Teh, Effect of stabilization temperature on the degradation of adriamycin in albumin microspheres, Biomat. Art. Cells Immob. Biotech., 19 (1991) 613-629.

10 C. Jones, M.A. Burton, B.N. Gray and J. Hodgkin, In vitro release of cytotoxic agents from ion exchange resins, J. Controlled Release, 8 (1989) 251-257.

11 W.E. Longo, H. Iwata, T. Lindheimer and E.P. Goldberg, Preparation and drug release properties of albumin-polyglutamic acid-adriamycin microspheres, Polymer Prep., 24 (1983) 56-57. 
12 N. Willmott, Y. Chen and A.T. Florence, Haemoglobin, transferrin and albumin/polyaspartic acid microspheres as carriers for the cytotoxic drug adriamycin. I. Ultrastructural appearance and drug content, J. Controlled Rclease, 8 (1988) 93-101.

13 H.F.M. Cremers, J. Feijen, G. Kwon, Y.H. Bae, S.W. Kim, H.P.J.M. Noteborn and J.G. McVie, Albuminheparin microspheres as carriers for cytostatic agents, $\mathrm{J}$. Controlled Release, 11 (1990) 167-179.

14 G.S. Kwon, Y.H. Bae, S.W. Kim, H. Cremers and J. Feijen, Preparation and characterization of microspheres of albumin-heparin conjugates, J. Colloid Interface Sci., 143 (1991) 501-512.

15 M. Menozzi and F. Arcamone, Binding of adriamycin to sulphated mucopolysaccharides, Biochem. Biophys. Res. Commun., 80 (1978) 313-318.

16 E. Cofrancesco, A. Vigo and E. Pogliani, Antiheparin activity of adriamycin, Thromb. Res., 18 (1980) 743746.

17 W.E. Hennink, J. Feijen, C.D. Ebert and S.W. Kim, Covalently bound conjugates of albumin and heparin: synthesis, fractionation and characterization, Thromb. Res., 29 (1983) 1-13.

18 H.F.M. Cremers, G.S. Kwon, Y.H. Bae, S.W. Kim, R. Verrijk, H.P.J.M. Noteborn and Feijen J., Preparation and characterization of albumin-heparin microspheres, Biomaterials (1994) accepted for publication.

19 G.S. Kwon, Y.H. Bae, H. Cremers, J. Feijen and S.W. Kim, Release of macromolecules from albumin-heparin microspheres, Int. J. Pharm., 79 (1992) 191-198.

20 R. Gillett and K. Gull, Glutaraldehyde: its purity and stability, Histochemie, 30 (1972) 162-167.
21 E. Hayakawa, K. Furuya, T. Kuroda, M. Moriyama and A. Kondo, Viscosity study on the self-association of doxorubicin in aqueous solution, Chem. Pharm. Bull., 39 (1991) 1282-1286.

22 S.R. Martin, Absorption and circular dichroic spectral studies on the self-association of daunorubicin, Biopolymers, 19 (1980) 713-721.

23 M. Nukui, C. Hoes, H. van den Berg and J. Feijen, Association of macromolecular prodrugs consisting of adriamycin bound to poly ( $\alpha$-L-glutamic acid $)$, Makromol. Chem., 192 (1991) 2925-2942.

24 F. Hellferich, Ion-exchange kinetics. In Marinsky, J.A. (Ed.) Ion Exchange, Vol 1, Marcel Dekker Inc., New York, 1969, pp. 135-166.

25 J. Crank, The Mathematics of Diffusion, Oxford Univ. Press, London, 1956.

26 R.W. Korsmeyer and N.A. Peppas, Effect of the morphology of hydrophilic polymeric matrices on the diffusion and release of water soluble drugs, J. Membrane Sci., 9 (1981) $211-227$.

27 R. Baker, Diffusion controlled systems. In: R. Baker, Controlled release of biologically active agents, J. Wiley \& Sons, New York, 1987, pp. 39-83.

28 P.L. Ritger and N.A. Peppas, $\Lambda$ simple equation for description of solute release I. Fickian and non-Fickian release from non-swellable devices in the form of slabs, spheres, cylinders or discs, J. Controlled Release, 5 (1987) 23-36.

29 M. Donbrow, A. Hoffmann and S. Benita, Variation of population kinetics in polydisperse multiparticulate systems (microcapsules, microspheres, droplets, cells) with heterogeneity of one, two or three parameters in the population of individuals, J. Pharm. Pharmacol., 40 (1988) 93-96. 